\title{
Aspectos bioéticos del consumo de alimentos genéticamente modificados (AGM)
}

\author{
Bioethical Aspects of Genetically Modified Food Consumption
}

Nancy G. Buendía-Sarabia ${ }^{a}$, Marcos M. Galván-García ${ }^{b} \&$ Guadalupe López-Rodríguez $^{c}$

\begin{abstract}
:
The effects of technological advancement of genetic engineering and molecular biology are both positive and negative. In its early life, genetically modified foods were intended to gain advantages in the areas of agriculture and livestock. This technique subsequently began to be applied in the field of food production for human consumption, generate a lot of controversy regarding its use. This work aims to review available scientific information regarding the use, benefits and potential risks to human health and the environment of the consumption of genetically modified foods, as well as their relationship to bioethics aspects.
\end{abstract}

Keywords:

Biotechnology, genetically modified foods, bioethics, human health.

\section{Resumen:}

Los efectos del avance tecnológico de la ingeniería genética y la biología molecular son tanto positivos como negativos. En sus inicios, los alimentos genéticamente modificados tenían la intención de obtener ventajas en las áreas de agricultura y ganadería. Esta técnica posteriormente comenzó a aplicarse en el campo de la producción de alimentos para el consumo humano, generando una gran controversia con respecto a su uso. Este trabajo tiene como objetivo revisar la información científica disponible sobre el uso, los beneficios y los riesgos potenciales para la salud humana y el medio ambiente del consumo de alimentos genéticamente modificados, así como su relación con los aspectos bioéticos.

\section{Palabras Clave}

Biotecnología, alimentos genéticamente modificados, inocuidad, bioética, salud humana.

\section{Introducción}

La biotecnología, es una ciencia multidisciplinaria que utiliza sistemas biológicos y organismos vivos o sus derivados para el desarrollo de nuevos productos o procesos para usos específicos. El hombre fue desarrollando actividades específicas para perfeccionar el conocimiento biotecnológico, desencadenando una necesaria reflexión ética y moral.

Dicha ciencia aplicada a los alimentos, no sólo tiene que ver con aumentar la producción de estos, o bien mejorarlos nutricionalmente, sino que también pretende atender la demanda de los consumidores, proporcionando alimentos más frescos, seguros, inocuos y con mayor vida de anaquel. Se reconoce que la biotecnología puede contribuir a elevar la producción y productividad alimentaria, a través de la implementación de marcos normativos para los ámbitos de la bioseguridad e inocuidad de los alimentos. Sin embargo, también se encuentran los posibles riesgos que la biotecnología moderna puede plantear, entre ellos los efectos sobre la salud humana y las consecuencias para el medio ambiente, de ahí que se generan grandes conflictos morales. Pero si se combina la ética con la manipulación adecuada de la producción de alimentos genéticamente modificados (AGM), la biotecnología podría ofrecer una alternativa para satisfacer las necesidades de una población cada vez más numerosa y más urbanizada, además de proporcionar beneficios para la salud humana [1]. 


\section{Hidalgo, Vol. 8, No. 15 (2019) 7-14}

El uso de la biotecnología para el desarrollo de AGM, trae varios beneficios, ya que dichos alimentos cuentan con una mayor calidad y valor nutricional, muchos de estos considerados incluso como alimentos funcionales. La información científica pretende garantizar a la sociedad que la biotecnología aplicada a la producción de alimentos, puede contribuir a la salud humana de manera segura. Alimentos que son mejorados genéticamente, tienen características favorables a las necesidades agronómicas, como son verduras con mayor resistencia al transporte y almacenamiento, e incluso más resistentes a las plagas [2].

La sociedad desempeña un papel relevante en el debate de las cuestiones éticas y en la confianza hacia la ciencia y la investigación. Actualmente, con el creciente desarrollo de este tipo de alimentos, ha existido controversia entre la sociedad acerca, especialmente, de los riesgos que los productos pueden acarrear, por lo que es importante esclarecer la información acerca de los AGM. Este ensayo tiene por objetivo aportar información científica actualizada sobre las aplicaciones, ventajas, riesgos para la salud humana y el medio ambiente de los AGM, con la finalidad de generar una discusión de los aspectos bioéticos que deben ser considerados para asumir una postura sobre ellos.

\section{Alimentos Genéticamente Modificados}

Según la Organización Mundial de la Salud (OMS), se definen como organismos en los cuales el material genético (ADN) ha sido modificado. Los AGM, son seguros para el medio ambiente y para el consumo humano, incluso pueden ser similares a los alimentos convencionales.

La aplicación de la biotecnología en la producción alimentaria permite que plantas y animales sean genéticamente modificados, con características que no son posibles mediante las técnicas de producción tradicional, y que incluso desde la perspectiva agrícola, contribuye a un aumento en la producción, así como un aumento en la calidad y mejorar las características nutricionales [3].

Estos alimentos producidos mediante la biotecnología suelen dividirse en 4 categorías: 1) Alimentos compuestos por lo que contienen organismos vivientes/viable, un ejemplo de ellos es el maíz. 2) Alimentos derivados $o$ que contengan ingredientes derivados de organismos genéticamente modificados (OGM), un ejemplo de este es la harina. 3) Alimentos que contengan un sólo ingrediente o aditivo producido por OGM, como colorantes, vitaminas y aminoácidos esenciales. Y 4) Alimentos que contengan ingredientes procesados por enzimas producidas por OGM, como el jarabe de maíz de alta fructuosa producido a partir del almidón, usando la enzima glucosa isomerasa [4].

\section{Beneficios de los AGM}

Durante los últimos 5 ○ 6 años, se ha generado una controversia en relación con los riesgos y beneficios para salud humana del consumo de los alimentos genéticamente modificados. Esto ha llegado incluso a las esferas socioeconómicas y legales, incrementándose notablemente en los últimos años. Sin embargo, el número de estudios científicos sobre los riesgos toxicológicos y efectos adversos sobre la salud del potencial consumo humano de los AGM, es muy escaso [5].

Los AGM cuentan con beneficios indirectos, como la reducción del uso de sustancias químicas para la agricultura, así como la sostenibilidad de los cultivos y la seguridad alimentaria para los consumidores; sin embargo, dichos alimentos pueden acarrear problemas directos para la salud y el desarrollo humano, debido a que algunos de los genes utilizados para modificar los alimentos carecen de antecedentes inocuos, por lo que existen acuerdos y normas que regulan estos alimentos antes de su comercialización. Algunos de los beneficios de los AGM es la producción masiva, que genera costos más bajos que un alimento no modificado, además de que dichos productos son más resistentes y su maduración es mucho más lenta que los alimentos tradicionales, por lo cual, permite alargar la vida de anaquel y mantener la calidad de los alimentos.

La mala distribución de los recursos y de los alimentos que se producen en el mundo, dan lugar a situaciones de hambre en países de bajos ingresos, que, además, implica aspectos económicos y sociales, pero que comprometen el desarrollo y vida de sus comunidades. Transformar los sistemas agrícolas de los agricultores rurales introduciendo tecnologías que integren procesos agroecológicos en la producción alimentaria mientras se minimizan los efectos adversos para el medio ambiente, es fundamental para la agricultura sostenible, que puede llegar a transformar la situación de carencia de alimentos de diversas partes del mundo [4].

La realización de pruebas dentro del ámbito alimentario en lo que respecta a la mejoría de dichos alimentos nutrimentalmente hablando, ha tenido resultados positivos, teniendo como principal beneficio el incremento de macronutrientes y micronutrientes mejor color, sabor y textura del alimento, así como la optimización de su duración y conservación.

De igual manera, el desarrollo de técnicas de manipulación genética puede ser usado para aumentar, disminuir o modificar la cantidad de nutrientes específicos de diferentes alimentos. Por ejemplo, existe un arroz genéticamente modificado, llamado "arroz dorado", que tiene incorporados 7 genes de distintos vegetales, que le confieren un mayor contenido de betacaroteno y de hierro ( $\mathrm{Fe}$ ), útiles para la prevención y manejo de la ceguera nocturna y anemia, patologías que son endémicas en algunas zonas del mundo. Con $300 \mathrm{~g}$ de dicho arroz, se logra cubrir el $50 \%$ de los 


\section{Hidalgo, Vol. 8, No. 15 (2019) 7-14}

requerimientos diarios de vitamina $\mathrm{A}$ y el $50 \%$ de los de Hierro (Fe) de adultos [6].

\section{Desventajas de los alimentos genéticamente modificados.}

Los AGM, también pueden tener impactos negativos sobre el medio ambiente e incluso sobre factores económicos, como el comercio local, factores sociales y factores bioéticos [3].

Los potenciales riesgos a los que nos podríamos ver expuestos con los AGM y que son el fundamento de organizaciones ecologistas que rechazan la utilización y consumo de AGM, son el desarrollo de alergias, la resistencia a los antibióticos, la pérdida o modificación del valor nutricional de los alimentos, la presencia de compuestos tóxicos, la aparición de enfermedades nuevas y no tratables, además del daño a las especies silvestres de plantas.

Se han producido diversas controversias nacionales e internacionales con respecto a la inocuidad de estos alimentos, puesto que estos alimentos han producido alergias en personas susceptibles. Esto es debido a que los genes, que es lo que se transfiere de un organismo a otro para obtener AGM, codifican ciertas proteínas que pueden ser alergénicas para un grupo de la población, por lo que en dichas personas o grupos se ha tomado como un riesgo el consumir alimentos genéticamente modificados más que el beneficio que se puede llegar a tener nutrimentalmente.

Las alergias a los alimentos se clasifican como:

- Clase 1 si la sensibilización ocurre en el tracto gastrointestinal.

- Clase 2 si ocurre a través de la inhalación del alérgeno

La población adulta presenta reacciones al maní, las nueces, el pescado y los mariscos explican la mayoría de estas reacciones. A estos alimentos se puede añadir una lista que incluye la soya, el trigo, el arroz, el maíz, el banano, el fríjol, el apio, el kiwi, la oliva, la papaya, la piña, los cítricos, la manzana, el tomate, entre otros; todos ellos modificados genéticamente (14)

Sin embargo, no existe evidencia suficiente de que se puedan transferir estos genes de resistencia desde los AGM al tracto digestivo humano. La posibilidad de que se transmita resistencia a los antibióticos a través del consumo de AGM, constituye uno de los mayores temores en relación a su ingesta, debido a que al utilizar bacterias $u$ otros microorganismos resistentes a un determinado antibiótico e ingerirlos, estos productos transmitirán la resistencia al antibiótico, lo que dificultará el manejo de patologías.

La presencia de toxinas en AGM se controla mediante lineamientos específicos, ya que en el caso de que las toxinas presentes sobrepasen la cantidad en el alimento original, el producto modificado no puede comercializarse. Además, cabe destacar que, en ocasiones, los objetivos de la biotecnología se basan precisamente en la reducción o eliminación de toxinas del alimento original, buscando el mayor beneficio a la salud poblacional.

Actualmente, no se tiene evidencia de que estos alimentos constituyan un riesgo potencial a la salud humana [7]. La mayoría de los estudios se han realizado en animales y por un plazo de 90 días únicamente, este tiempo es muy poco si se busca determinar un posible efecto adverso a largo plazo. Sin embargo, existen estudios donde se han reportado casos de cáncer en ratas que han sido alimentadas con AGM [14]. Será importante llevar cabo investigaciones sobre los efectos adversos que pueda tener el consumo en humanos al largo plazo [7].

\section{Aspectos sociales y económicos}

Es necesario evaluar el impacto que tendrían los AGM a nivel mundial en el problema de inseguridad alimentaria. Por un lado, no cabe duda de los potenciales beneficios con esta tecnología, y por otro lado se debe considerar cómo se absorbería la desocupación campesina que se generaría al no necesitar fuerza de trabajo para desmalezar siembras, ya que ahora son resistentes a plagas y malezas, o cómo se solucionaría el impacto económico causado al utilizar genes que codifican características singulares de un producto que es la base de la economía de una región o país. El impacto en la biodiversidad, disminuye la capacidad de respuesta frente a cambios ambientales que pongan en riesgo los cultivos de producción.

Se deben definir los límites éticos para la manipulación de genes y la protección de zonas o países que, al no contar con los recursos y tecnología para desarrollar y explotar su propio potencial, pueda ser utilizado y patentado por quien posee las herramientas y capital, generando conflictos de desplazamiento de pequeños productores, de propiedad y patentes similar al generado en la industria farmacéutica. Sin duda se debe normar y legislar, para lograr utilizar la técnica de forma cuidadosa, responsable y transparente.

\section{Aspectos bioéticos del consumo de AGM}

Abordar el tema de los AGM desde el punto de vista de la bioética, puede ayudar a tomar una postura sobre su utilización en el consumo humano y asumir una postura frente a ello. Una reflexión bioética puede contribuir a apoyar o rechazar la producción de alimentos genéticamente modificados; sin embargo, el debate público sobre dicho tema se encuentra limitado por la falta de conocimiento y la propia ideología o religión. Los argumentos entre la aprobación o rechazo de estos alimentos se basa en perspectivas éticas ampliamente diferentes, para algunos la modificación genética es inmoral, por afectar el valor intrínseco de los seres vivos, 
de forma que las preocupaciones éticas y morales son factores importantes que influyen en el temor de consumidor sobre los posibles riesgos que puede ocasionar la producción de AGM [8].

La reflexión bioética tiene la ventaja de permitir el intercambio de opiniones entre expertos y no expertos. Favoreciendo el diálogo en la medida en que los individuos interesados profundicen en los temas genómicos y sus implicaciones sociales, en especial sobre los beneficios y riesgos de los AGM basados en evidencia científica y no así en creencias o mitos, clasificando lo artificial como malo o dañino. Los argumentos a favor y en contra del uso de organismos genéticamente modificados (OGM) se basan en visiones de la nueva tecnología desde perspectivas éticas ampliamente diferentes, si bien los seres humanos tienen un gran poder sobre la naturaleza gracias a la biotecnología, que permite alterar y manipular en gran medida la vida, lo que requiere regulación para poder tener un control en su ejercer. Es por eso que éticamente hay que evitar una concepción utilitaria de la naturaleza, estableciendo límites en la actuación humana sobre ella para evitar que se considere a los seres vivos como meros objetos susceptibles de apropiación [9].

Es positiva la investigación e innovación que se ha realizado en cuanto a las patentes de organismos genéticamente modificados; sin embargo, los cuestionamientos apuntan a que básicamente se trata de intereses económicos de grandes corporativos, sin dar debida importancia a temas de sostenibilidad en agricultura y ganadería, salud y nutrición humana, ni haber consultado a las comunidades que se sienten agraviadas. En la reflexión bioética existe una considerable controversia en cuanto al valor de los AGM o transgénicos como también se le conocen, porque éstos pueden afectar el equilibrio ecológico, la biodiversidad y la economía local, además de cuestionar que el valor intrínseco de un ser vivo puede quedar afectado al ser sujeto de modificación genética, lo cual contraviene el manejo bioético de las especies, e incluso el equilibro económico de comunidades tradicionales. Entrando en el dilema de cómo conservar sin destruir lo que la naturaleza le ha llevado miles de años en desarrollar.

Dentro de las aplicaciones en la agricultura se encuentra el mejoramiento en calidad nutricional o rendimiento, por ejemplo, el incremento de cosechas de arroz con plantas más cortas que producen más nutrientes disponibles para la producción de grano [10]; así como la alteración del tiempo para retardar la maduración y contar con más tiempo para el transporte y almacenamiento. Otro ejemplo es la maduración retardada del tomate hace que se mantenga firme por mucho tiempo después de la cosecha. El tomate tradicional es cosechado mientras está todavía verde y firme para que no se dañe durante el transporte al supermercado y es rociado con etileno después del transporte para inducir la maduración [11]. Aunque es poco probable que el etileno permanezca en los alimentos, no se tiene toda la certeza dependiendo de la concentración; estudios en animales han demostrado que respirar óxido de etileno a altos niveles puede afectar la capacidad de reproducir. Lo anterior ilustra que aún las prácticas del manejo tradicional de ciertos productos para el consumo humano tienen implicaciones éticas que deberían revisarse.

La aplicación de los principios bioéticos puede ayudar a tomar medidas sobre la racionalidad del uso de transgénicos y otras consecuencias, existiendo la necesidad de regulaciones que controlen su producción en los países. De ahí que el principio bioético de "no maleficencia", en el caso de los AGM en muchos casos no se cumple, ya que se tiene poca evidencia científica sobre las consecuencias que puede traer la introducción no controlada de un alimento genéticamente modificado, ya sea de forma directa o indirecta. En cuanto al principio bioético de "beneficencia", se debe buscar que la producción de AGM sea accesible en cuanto precio para las personas de bajos recursos para que puedan hacer uso de estos productos, especialmente en el caso de poblaciones de países que no tienen acceso a alimentos con mayor aporte nutrimental; sin embargo, y de acuerdo a un informe del 2018 de la Organización de las Naciones Unidas para la Agricultura y la Alimentación (FAO), aún existen en el mundo 821 millones de personas que padecen hambre y más de 150 millones de niños sufren retraso del crecimiento. Por otra parte, para cumplir con el principio bioético de "autonomía" los consumidores tienen el derecho y deben estar enterados que los alimentos que consumen son genéticamente modificados para tomar una decisión, si los alimentos no están etiquetados o bien no tienen una leyenda, se estaría violando el derecho del consumidor. En varios países no existe una ley que obligue a la industria a declarar de forma explícita y clara el contenido en los alimentos para consumo humano de OGM [8]., sin embargo, en México se cuenta con una ley que regula el ejercer sobre este tema "Ley De Bioseguridad De Organismos Genéticamente Modificados" [15]. Finalmente, el principio bioético de "justicia" plantea la distribución equitativa de los beneficios de la producción de OGM, pero esto está muy lejos de alcanzarse, ya que la industria protege mediante patentes sus nuevos alimentos, lo que origina que no se compartan de forma apropiada los beneficios. [8].

Para hacer énfasis en el principio de "justicia" (dar a cada uno lo que necesita) en el tema de los OGM, se debería salvaguardar la protección de poblaciones vulnerables buscando una equidad en el uso de tecnología, es decir, se plantea la distribución equitativa de los beneficios, garantiza una justicia intergeneracional respecto del cuidado del ecosistema y la distribución de recursos fruto del trabajo colectivo; y establece una justicia ambiental en cuanto a penalizar a los agentes de contaminación ambiental. En este principio se halla enmarcado el tema de la 
responsabilidad hacia generaciones futuras como desafío que necesita de una reflexión bioética. La responsabilidad significa realizar un balance entre riesgos y beneficios de los OGM en el desarrollo social [9]. Es decir, si los AGM cumplen con el principio de "equivalencia sustancial" que lo caracterizaría como equivalente a su predecesor convencional, lo cual ocurre sólo cuando se presume que no genera nuevos riesgos $y$, por lo tanto, es aceptable para el consumo humano. [12] Pero de esto no existe suficiente evidencia en el largo plazo.

La introducción de la tecnología de AGM o transgénicos, debe hacerse considerando la participación de agricultores, ganaderos y consumidores, y no guiarse simplemente por intereses políticos y comerciales de empresas trasnacionales. Existen temas de sostenibilidad a largo plazo y riesgos ecológicos que deben contemplarse, teniendo en cuenta los principios de precaución y responsabilidad hacia las generaciones futuras, y estos es lo que se debería tomar en cuenta en primer lugar.

Es necesario que exista monitoreo y evaluación de riesgos ambientales y sociales de los productos de la biotecnología. Ciertas áreas deberían protegerse para tener solo cultivos orgánicos, y la ley de bioseguridad aplicada a transgénicos debe tener en cuenta todos los elementos para disminuir riesgos. No etiquetar que un alimento contiene un porcentaje transgénico viola el derecho de los consumidores a saber, y esto es muy grave porque puede poner en riesgo su salud si es que el consumidor final presenta una alergia o una reacción adversa a estos alimentos.

\section{Las cargas de salud conocidas o potenciales de los AGM}

La maleficencia es de mayor preocupación con la tecnología de AGM, en particular porque todavía faltan investigaciones que determinen el alcance de efectos adversos. Cerca de 36 países, incluyendo a más de la mitad de la Unión Europea, han prohibido realizar cultivos transgénicos debido a su potencial riesgo de causar daño. La preocupación de los daños que pueden ocasionar los AGM ha sido interpretada para justificar la imposición de una prohibición absoluta sobre la tecnología de AGM, aunque es casi imposible para cualquier científico prometer riesgo absoluto independientemente de la tecnología de alimentos propuesta. Otra carga potencial de la tecnología de AGM es cómo la tecnología compite con estrategias alternativas, incluyendo, diversidad de la cultura alimentaria y la fortificación.

Un futuro riesgo hipotético, es que los alimentos genéticamente modificados dominen la canasta básica de alimentos, lo que conlleva a que el mercado se centre menos en otras fuentes de alimentos ricos en nutrientes, por ejemplo, los cultivos de los pequeños productores quienes no cuentan con los recursos e infra estructura para producir AGM y que por consecuencia podemos decir que son más naturales, pero al mismo tiempo requieren de un mayor tiempo de producción siendo esto contrario a lo que se busca al cultivar los AGM, más allá de los granos básicos. Los cultivos transgénicos también pueden crear un riesgo adicional de desplazar a las variedades de cultivos tradicionales que tienen un mayor valor nutritivo y se adaptan localmente al medio ambiente. Independientemente, los AGM no deben ser descontados inmediatamente como una herramienta para asegurar una diversidad alimentaria.

Desde el punto de vista de la nutrición, actualmente no hay evidencia de que los alimentos genéticamente modificados sean una amenaza para la salud comparado con los cultivos convencionales. Sin embargo, existe una preocupación que refleja los riesgos para la salud en la producción que surgen con los cultivos transgénicos orientados a los agricultores (primera generación). Si bien la tecnología de AGM no plantea un riesgo para la salud en la medida en que las pruebas sugieren, los plaguicidas y los herbicidas utilizados para cultivar alimentos transgénicos de primera generación podrían colocar a los trabajadores agrícolas y a los consumidores en un riesgo mayor e indebido, ya que existe evidencia sobre el riesgo de cáncer y productos químicos específicos, como el glifosato. Desde el punto de vista de la nutrición en salud pública, los beneficios de la tecnología AGM de segunda generación podrían compensar los riesgos, especialmente para las poblaciones que sufren de malnutrición por micronutrientes con poco acceso a diversos alimentos de calidad. Sin embargo, en lugares donde la tecnología AGM requiere más uso de plaguicidas, los riesgos desconocidos para la salud de la población deben ser examinados en profundidad para evaluar los compromisos éticos en referente al cultivo y consumo de los mismos.

Es importante considerar tanto el contexto en el que se están cultivando estos alimentos como la población a la que pueden beneficiar estos alimentos, a fin de reducir al mínimo los riesgos a la salud. Los cultivos diseñados con deficiencias de micronutrientes pueden evaluarse de manera diferente que los obtenidos para los atributos sensoriales o promotores de la salud, ya que el contexto de uso de AGM es distinto. Aunque, por otro lado, los cultivos transgénicos de segunda generación criados con rasgos sensoriales o atributos de promoción de la salud están enfocados a la población en general, sin poner la suficiente atención al valor nutrimental, lo anterior, derivado de los mismos intereses del consumidor, así como la población objetivo a la que se dirige o por la cual se diseña la producción de AGM.

Sin embargo, sigue existiendo mucha información negativa que a menudo domina las opiniones de los consumidores, incluso cuando se combina con la información positiva, dado que se realiza un juicio generalizado de este tipo de productos, aunado a la falta de información acerca de sus beneficios. 
Las percepciones de riesgo de los alimentos genéticamente modificados también varían según la región, como es el caso de los consumidores en Europa, que tienen más percepciones y actitudes negativas para comprar alimentos genéticamente modificados en comparación con los norteamericanos que tienen una mayor aceptación a este tipo de alimentos, mientras que en México existe un argumento emitido por aquellos que están en contra de estos alimentos, plantea que su cultivo representa un riesgo para la biodiversidad del maíz y otras especies endémicas del país [16].

Por lo cual, en lo que se refiere a la información y la comunicación acerca de los alimentos genéticamente modificados, la comunidad científica y las industrias de semillas pueden desempeñar un mejor papel respondiendo a las preocupaciones, preguntas y limitaciones a las que se enfrenta la aceptación de los AGM por la población en general, lo que puede dar paso a solucionar los problemas que son en la gran mayoría de los casos, los causantes de la producción de AGM, especialmente aquellos que se refieren a la situación de hambre a nivel mundial.

En lo que respecta al costo monetario de estos productos, la disposición de los consumidores a pagar por los alimentos genéticamente modificados, está positivamente correlacionada con los atributos nutricionales, hablando del caso de grupos sociales que cuentan con los recursos económicos suficientes. Esto sucede cuando los fabricantes proporcionan una comunicación clara acerca de la composición y beneficios que puede adquirir el consumidor, situación que puede recomendarse para todos los productores de estos alimentos, para generar información correcta y conocimiento de los AGM a la población.

En los países donde las deficiencias de micronutrientes son el objetivo de una tecnología intermedia, llamar la atención sobre la malnutrición y los beneficios de la tecnología junto con otras estrategias puede ayudar a la toma de decisiones. El comportamiento del consumidor local, las percepciones y las actitudes deben ser mejor comprendidos con el fin de resolver conflictos existentes en relación con los AGM [13].

\section{CONCLUSIÓN}

El avance en biotecnología ha supuesto la solución a diversos problemas, entre los que se encuentran la desnutrición infantil de países de bajos ingresos. Se ha buscado mejorar las cualidades de los alimentos, desde el punto de vista nutricional y sensorial. Con el continuo desarrollo de nuevas tecnologías que dan lugar a este tipo de productos, se ha desarrollado también el interés de la sociedad acerca de cuáles son los lineamientos que rigen la producción de alimentos genéticamente modificados, lo que resulta clave en el cumplimiento de las normativas vigentes, dado que es el consumidor quien está interesado en la procedencia y procesos que se llevan a cabo para llevar los alimentos a su mesa. Sin embargo, este aparente interés ha tenido también un impacto negativo, dado que existen prejuicios y creencias diversas acerca de que gran cantidad de AGM representa un riesgo potencial a la salud humana, a la biodiversidad y al medio ambiente. El papel del nutriólogo, como profesional de la salud que busca mejorar la calidad de vida de la población a través de dietas más saludables, es mantenerse informado de fuentes confiables basadas en evidencia científica sobre la diversidad de AGM que llenan el mercado en la actualidad, dado que es casi inevitable toparse con algún alimento que haya sufrido alguna intervención biotecnológica. De esta forma, podremos informar a la población sobre las ventajas de estos alimentos, y prevenir el consumo de productos que no cumplan con especificaciones en su producción, protegiendo así la salud de la población, y determinando cuáles son los productos que podemos utilizar, especialmente tratándose de personas con requerimientos 0 deficiencias específicas. Además de realizar campañas de comunicación en salud y realizar a través de organismos colegiados la solicitud de mejoras de las leyes y reglamentos que regulan la producción, consumo y publicidad de alimentos genéticamente modificados. 


\section{Figura 1. Mapa beneficios y efectos nocivos de los alimentos genéticamente modificados}

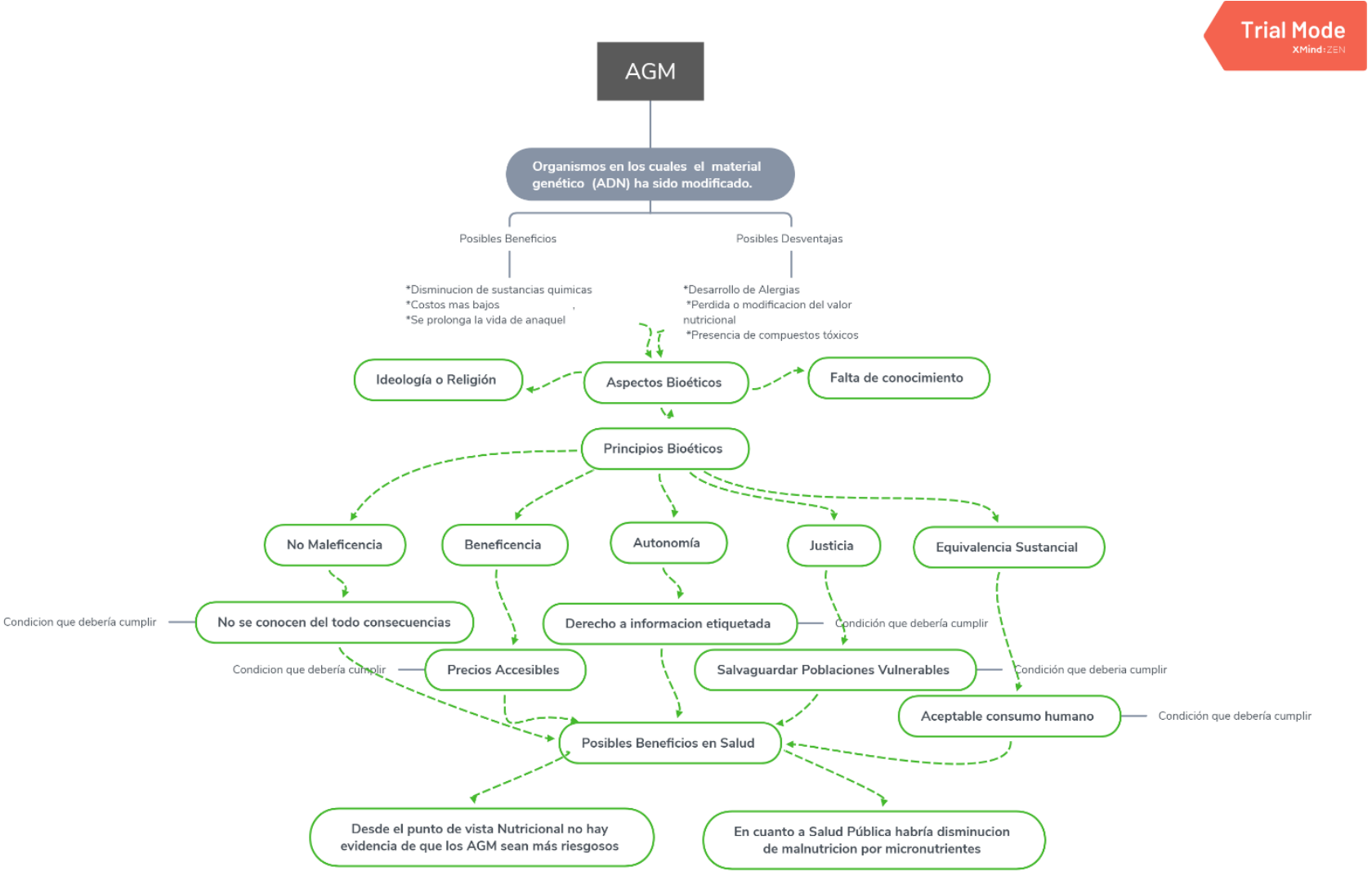

\section{Referencias}

1. Dilla FAO: Organización de las Naciones Unidas para la Alimentación y la Agricultura [Internet]. FAO 2009. [citado 16 May 2017]. Disponible en:http://www.fao.org/biotechnology/es/

2. Beraldo D, Endres L, Amaral B. Biotecnología aplicada a la alimentacion y salud humana. Rev Child Nutr 2012;39;94-98

3. OMS: Organización Mundial de la Salud [Internet].Suiza:OMS 2005. [citado 16 May 2017]. Disponible en: http://www.who.int/foodsafety/publications/biotech/biotech_sp .pdf

4. OMS. Biotecnología moderna de los alimentos, salud y desarrollo humano: estudio basado en evidencias. Ediciones de las OMS; 2015.

5. Domingo J, Gomez M. Riesgos sobre la salud de los alimentos genéticamente modificados. Rev Esp Salud Pública 2000; 74; 255-261.

6. Ye X, Al-Babili S, Kloti A, Zhang J, Lucca P, Beyer P, Potrykus I. Engeering the provitamin A (Beta- carotene) biosynthetic pathway into rice endosperm. Science 2000; 287 (5451): 303-5.
7. Safety of Genetically Engineered Crops. VIB Publication Flanders Interuniversity Institute for Biotechnology. March 2001:27-135

8. Rodríguez E. Temas éticos en investigación internacional con alimentos transgénicos. Rev. Act. Bioeth. 2013; 19 (2):209-218

9. Rodríguez E. Reflexión bioética sobre el uso de organismos genéticamente modificados. Rev. Bioethikos. 2010; 4 (2): 222227.

10. Hefferon KL. Recent patents in plant biotechnology: impact on global health. Recent Patents Biotechnology 2012; 6(2): $97-$ 105. PMID: 22642820

11. Davies HM. Review article: commercialization of whole-plant systems for biomanufactoring of protein products: evolution and prospects. Plant Biotechnology Journal 2010; 8(8): 845861. PMID 20731788.

12. ONU, FAO. Acuerdo de la OMS y la Food and Agriculture Organization, organismo dependiente de las Naciones Unidas; 1990.

13. Glass S, Fanzo J. Genetic modification technology for nutrition and improving diets: an ethical perspective. Elsevier. Current Opinion in Biotechnology 2017; 44:46-51. 
Publicación semestral, Educación y Salud Boletín Científico Instituto de Ciencias de la Salud Universidad Autónoma del Estado de

Hidalgo, Vol. 8, No. 15 (2019) 7-14

14. Acosta O., Guerrero C., Alimentos transgénicos y alergenicidad. Revista de la Facultad de Medicina, (2007), 55(4), 137, 251-269. Recuperado de https://revistas.unal.edu.co/index.php/revfacmed/article/view/2 3088/63905)

15. Diario Oficial de la Federación DOF 18-03-2005, Ley De Bioseguridad De Organismos Genéticamente Modificados, 2005 .

16. Montesinos O, Franco E, Luna I, A. F. (2016). Percepciones y actitudes hacia los transgénicos en México. México: Pearson. 